\title{
Fibroblast Growth Factor-23 Secreting Tumour Induced Osteomalacia
}

Economos $\mathrm{H}^{1 *}$, Bonar $\mathrm{F}^{2}$ and Diamond $\mathrm{T}^{3}$

${ }^{1}$ Department of Endocrinology, St George Hospital, Australia

${ }^{2}$ Depatment of Histopathology, Douglass Hanly Moir Pathology Maquarie Park, Australia

${ }^{3}$ Department of Endocrinology, St George Hospital, University of New South Wales, Australia

*Corresponding author: Economos H, Department of Endocrinology, Level 3 Pritchard Wing, St George Hospital, Short St, Kogarah, NSW 2217, Australia

Received: May 21, 2021; Accepted: J une 24, 2021; Published: J uly 01, 2021

\begin{abstract}
Tumour Induced Osteomalacia (TIO) caused by a Fibroblast-GrowthFactor-23 (FGF-23) secreting tumour is a rare paraneoplastic disorder. Patients often present with non-specific complaints of pain and stiffness however findings of hypophosphataemia and inappropriately suppressed 1,25 dihidroxyvitamin $\mathrm{D}$ are almost pathognemonic of this condition. We present a previously healthy 51-year-old man who presented with an 18 month history of body aches, hypophosphataemia, and an incidental finding of atraumatic foot fractures on x-ray. Technetium bone scan identified diffuse skeletal pathologic fractures. He was further found to have persistent hypophosphataemia, suppressed 1-25-dihidroxyvitamin-D, renal phosphate wasting with reduced Tubular Reabsorption of Phosphate (TRP), and an FGF-23 level more than twice the upper limit of normal. Fluorodeoxyglucose (FDG) Positron Emission Tomography (PET), Gallium-68 Dotatate PET and magnetic resonance imaging further identified and characterised a $40 \mathrm{~mm}$ FDG and Dotatate-avid lesion in the right third rib. Histopathological examination of a biopsy specimen revealed phosphaturic mesenchymal tumour with positive FGF-23 immunohistochemical staining arising in a background consistent with osteomalacia. After tumour resection, the patient's hypophosphataemia, reduced TRP and elevated FGF23 normalised and he made an excellent recovery with rehabilitation. This case report illustrates the importance of a diagnosis of hypophosphataemia in a patient with musculo-skeletal aches and alerts clinicians of the differential diagnosis of TIO.
\end{abstract}

Keywords: Hypophosphataemia; FGF-23; Tumour induced osteomalacia; oncogenic osteomalacia

\section{Case Presentation}

A previously healthy 51 -year-old man was referred to a tertiary hospital by his general practitioner with an 18 month history of worsening musculoskeletal pains, chronic hypophosphataemia 0.43 $0.65 \mathrm{mmol} / \mathrm{L}$ (normal reference 1.12-1.45) and technetium whole body bone scan showing widespread pathologic skeletal fractures.

His pain was associated with progressive stiffness and generalised weakness. He reported that the severity of his symptoms, especially in his left hip, were impacting his mobility and forcing him to commence sick leave from his job as a boiler maker. An x-ray from a recent presentation for an infected foot laceration demonstrated multiple incidental fractures without a prior history of trauma. A subsequent technetium whole body bone scan confirmed multiple areas of tracer uptake corresponding to over 30 stress fractures including bilateral calcanei, distal tibial plafond, both midfeet, left lateral tibial plateau, left femoral neck, right acetabulum, multiple ribs, both scapulae, right ilium and multiple vertebral bodies.

The patient denied any known risk factors for osteoporosis. His medications on admission included intermittent use of paracetamol and ibuprofen as needed. There was no history of alcohol or substance abuse and he was a non-smoker. He was previously healthy and active.

Physical examination was unremarkable apart from his extensive bony tenderness of ribs, back, vertebrae, pelvis, and lower limbs.
Admission full blood profile, renal function, serum calcium (2.21 mmol/L), 25-hydroxyvitamin $\mathrm{D}(79 \mathrm{nmol} / \mathrm{L})$ and parathyroid hormone $(4.6 \mathrm{pmol} / \mathrm{L})$ were within normal range. Serum phosphate levels were repeatedly low $(0.49 \mathrm{mmol})$ and 1,25 dihydroxyvitamin D was suppressed (24 pmol/L, normal reference 60-200). A 24-hour urine collection demonstrated a renal phosphate leak with a low tubular reabsorption of phosphate (TRP) (68.8\%, normal reference $82-95 \%)$.

The patient was initially admitted under the Rheumatology Department and treated on high doses of oral phosphate (Sandoz phosphate $1000 \mathrm{mg}$ TDS) and calcitriol (Rocaltrol $0.5 \mathrm{mcg}$ TDS). His analgesia was uptitrated. During his admission, intravenous phosphate replacement was required due to his intolerance to oral preparations.

A differential diagnosis included Tumour Induced Osteomalacia (TIO) or adult onset Hereditary Hypophosphataemic Rickets (HHR). $\mathrm{He}$ was referred to the Endocrinology Department for further investigations. Fibroblast-growth factor 23 (FGF-23) was measured and found to be more than twice the upper limit of normal (201 ng/ $\mathrm{mL}$ normal reference 23.2-95.4) suggestive of TIO as the cause for the his phosphate wasting.

A Fluorodeoxyglucose (FDG) positron emission tomography (PET) scan identified a $40 \mathrm{~mm}$ FDG-avid lesion in the right third rib which was further characterised as Dotatate-avid (SUV 34.6) using 


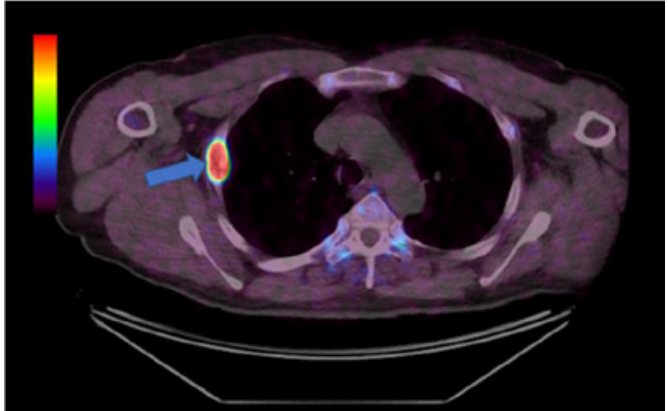

Figure 1: Fused axial dotatate scan view.

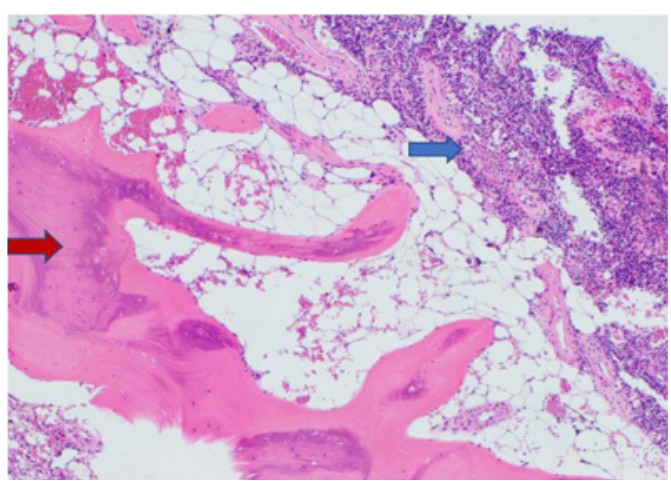

Figure 2: Phosphoturic mesenchymal tumour arising in a background of osteomalacic bone.

a more sensitive Gallium-68 Dotatate PET Computed Tomography (CT) (Figure 1, arrow). Magnetic resonance imaging scan of the rib demonstrated low signal intensity on T1 and Short-TI Inversion Recovery with internal heterogeneity and peripheral hyperintensity corresponding to a $40 \mathrm{~mm}$ expansile ovoid bony lesion in the peripheral portion of the right third rib.

CT guided bone biopsy was performed and histopathological examination confirmed features of a phosphaturic mesenchymal tumour with accompanying osseous trabeculae showing features consistent with osteomalacia (Figure 2, abnormal woven bone and increased osteoid: red arrow, mesenchymal tumour: blue arrow). Immunohistochemical staining showed positivity for FGF-23 (Figure 3). Partial rib resection with wide margins surrounding the tumour was performed by the Cardiothoracic Department and submitted for histological analysis.

The postoperative serum phosphate trended to normal $(1.12 \mathrm{mmol} / \mathrm{L})$ within 4 days. At two weeks after surgery, the repeat FGF-23 (59.3 ng/mL) and TRP (94\%) had normalised. The widespread bone pains remained persistent and required ongoing oral analgesia and eventually settled over 8 weeks. The patient attended for tertiary rehabilitation and returned to work at three months after his successful surgery.

\section{Discussion}

TIO is an important cause of chronic hypophosphataemia and needs to be differentiated from other causes of renal phosphate wasting such as primary disorders of the proximal convoluted tubules

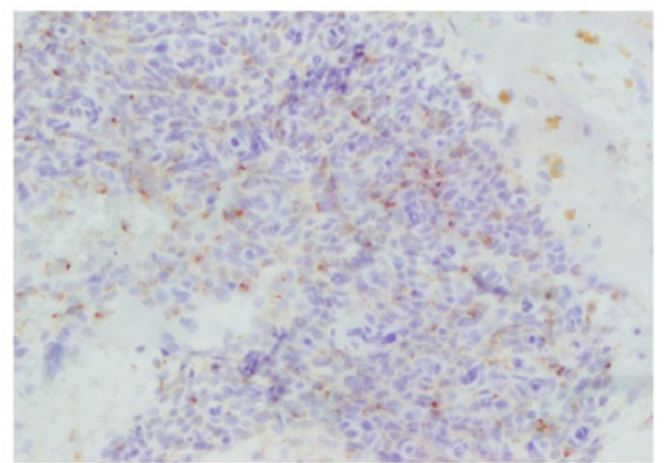

Figure 3: Immunohistochemical stain showing FGF 23 expression.

(Fanconi disease, amyloid or medications) or HHR (mutations in genes encoding for PHEX or FGF-23) [1]. In TIO, the leading culprit is usually a benign FGF-23 secreting mesenchymal tumour which rarely undergoes malignant transformation $[2,3]$. The findings of elevated serum FGF-23 and suppressed serum 1,25 dihydroxyvitamin D levels are pathognemonic for the condition [3-5]. HHR may have a similar presentation but serum FGF-23 and 1,25 dihydroxyvitamin D levels are usually normal $[1,6]$. There is often positive family history and a genetic panels for gene mutations (PHEX, FGF-23) are often required [6].

This case highlights the issues of diagnostic delay and inability to recognise the significance of hypophosphataemia in the clinical setting of non-specific musculo-skeletal pains and body aches. The profound myopathy and pain leading to a progressive disability is a frequent finding in patients presenting with TIO [7].

The bone pain is often related to the severity of the osteomalacia and pseudofractures, while the myopathy is due to the hypophosphataemia [1,3,7]. Osteoporosis is often diagnosed due to the low bone mass and increased osteoid [8]. Tumour resection leads to a decline in FGF-23 and reversal of the phosphate wasting [1]. Rapid healing of chronically unmineralized bone requires large amounts of calcium for bone accruel $[9,10]$. Our patient's serum calcium remained normal. There have been reports of cases of bone remineralisation mimicking hungry bone syndrome post FGF-23 tumour resection $[2,10]$.

\section{Conclusion}

This case provides an example of a patient with a prolonged prodrome of musculo-skeletal syndrome and biochemical of hypophosphatemia. He was fortunate to have a correct diagnosis considered after presenting with incidental multiple fractures. Detailed investigations led to a successful diagnosis, optimal treatment and an excellent outcome. This case emphasises the importance of diagnosing hypophosphataemia and the differential diagnosis which includes an FGF-23-secreting-tumour causing TIO.

\section{References}

1. Chong WH, Molinolo AA, Chen CC, Collins MT. Tumor-induced osteomalacia. Endocrine-Related Cancer. 2011; 18: R53-R77.

2. Kumar S, Diamond T. Lessons learnt from delayed diagnosis of FGF-23producing tumour-induced osteomalacia and post-operative hungry bone syndrome. Bone Reports. 2020; 12: 100276. 
3. Florenzano P, Gafni RI, Collins MT. Tumor-induced osteomalacia. Bone Reports. 2017; 7: 90-97.

4. Endo I, Fukumoto S, Ozono K, Namba N, Tanaka H, Inoue D, et al. Clinica usefulness of measurement of fibroblast growth factor 23 (FGF23) in hypophosphatemic patients: proposal of diagnostic criteria using FGF23 measurement. Bone. 2008; 42: 1235-1239.

5. Fukumoto S. FGF23-related hypophosphatemic rickets/osteomalacia: diagnosis and new treatment. Journal of Molecular Endocrinology. 2021; 66: R57-R65.

6. Bergwitz C, Miyamoto K-I. Hereditary hypophosphatemic rickets with hypercalciuria: pathophysiology, clinical presentation, diagnosis and therapy. Pflügers Archiv-European Journal of Physiology. 2019; 471: 149-163.

7. Minisola S, Peacock M, Fukumoto S, Cipriani C, Pepe J, Tella SH, et al
Tumour-induced osteomalacia. Nature Reviews Disease Primers. 2017; 3: 17044

8. Sood A, Agarwal K, Shukla J, Goel R, Dhir V, Bhattacharya A, et al. Bone scintigraphic patterns in patients of tumor induced osteomalacia. Indian J Nucl Med. 2013: 28: 173-175.

9. Jain N, Reilly RF. Hungry bone syndrome. Curr Opin Nephrol Hypertens 2017; 26: 250-255.

10. Rendina D, De Filippo G, Tauchmanovà L, Insabato L, Muscariello R, Gianfrancesco F, et al. Bone Turnover and the Osteoprotegerin-RANKL Pathway in Tumor-Induced Osteomalacia: A Longitudinal Study of Five Cases. Calcified Tissue International. 2009; 85: 293-300. 